American Journal of Applied Sciences 2 (10): 1389-1394, 2005

ISSN 1546-9239

(c) 2005 Science Publications

\title{
Flow of Dividends under a Constant Force of Interest
}

\author{
${ }^{1}$ Juma Kasozi and ${ }^{2}$ Jostein Paulsen \\ ${ }^{1}$ Department of Mathematics, Makerere University, Box 7062, Kampala, Uganda \\ ${ }^{2}$ Department of Mathematics, University of Bergen, 5008 Bergen, Norway
}

\begin{abstract}
This study addresses the issue of maximization of dividends of an insurer whose portfolio is exposed to insurance risk. The insurance risk arises from the classical surplus process commonly known as the Cramér-Lundberg model in the insurance literature. To enhance his financial base, the insurer invests in a risk free asset whose price dynamics are governed by a constant force of interest. We derive a linear Volterra integral equation of the second kind and apply an order four Block-byblock method of Paulsen et al. ${ }^{[1]}$ in conjunction with the Simpson rule to solve the Volterra integral equations for each chosen barrier thus generating corresponding dividend value functions. We have obtained the optimal barrier that maximizes the dividends. In the absence of the financial world, the analytical solution has been used to assess the accuracy of our results.
\end{abstract}

Key words: Risk theory, Volterra equation, block-by-block method, barrier strategy, dividends

\section{INTRODUCTION}

The model we consider in this study derives its name from the path breaking work of Lundberg ${ }^{[2]}$ and Cramer ${ }^{[3-5]}$. In this model, at time $t$, the surplus $Y_{t}$ of an insurance company evolves according to:

$$
Y_{t}=y+p t-\sum_{i=1}^{N_{t}} S_{i}
$$

where $y=Y_{0} \geq 0$ is the initial reserve, $p>0$ is the premium rate, $\left\{N_{t}\right\}_{t \in \mathfrak{R}^{+}}$is a Poisson process with intensity $\lambda$, modelling the number of claims (jumps) in $(0, t]$ and $\left\{s_{i}\right\}_{i \in N}$ is an independent and identically distributed sequence of positive random variables (with distribution $F$ ) independent of $N$, modelling the jump sizes. The distribution $F$ has finite expectation $\mu$ and finite variance $\sigma^{2}$.

The surplus process (1) with the risk averse condition that $p>\lambda \mathrm{E}\left(S_{1}\right)$ leads to the unrealistic situation $Y_{t} \rightarrow \infty$, as $t \rightarrow \infty$. Hence the need to modify the surplus process to take care of this. One way is to predetermine a surplus level $b$ in such a way that when the surplus level hits $b$, premium income is paid to shareholders as dividends. If the surplus is $b$ and a claim occurs, the claim is paid and no dividends are paid until the surplus hits $b$ again. Eventually and with certainty, ruin will occur at some stage in the future. When ruin occurs, the process stops. The question at hand is 'what $b$ ensures that the insurer pays maximum dividends to shareholders before ruin/bankruptcy?'
We concentrate on barrier strategies in this study although they may not be optimal when compounded by a diffusion in a vibrant financial market in which case other strategies e.g. b and strategies take an upper hand. Under the barrier strategy, we study the expected discounted dividend payments prior to ruin with $\delta$ as the discount factor when the model (1) is compounded by a constant force of interest. According to Miller Modigliani theory, this approach can be used as a valuation tool for companies, since the value of a company is exactly expected present value of future dividends ${ }^{[6]}$. Their theory was developed under the assumption of perfect capital markets, rational behavior and perfect certainty.

De Finetti ${ }^{[7]}$ underscored the importance of dividend payments in the economic considerations and management of insurance companies. His work culminated into a barrier strategy as being the optimal strategy. Dividend barrier models have a long history of risk theory ${ }^{[8-10]}$. The later work on optimal dividends was extensively discussed by Borch ${ }^{[11,12]}$. Gerber ${ }^{[13,14]}$ studied dividend payouts in (1) with the safety of the company in mind. Most literature is available in Gerber ${ }^{[15]}$ and the references therein. In many later papers, the problem is formulated and solved in the framework of the Brownian motion model, see e.g. Jeanblanc-Picqué and Shiryaev ${ }^{[16]}$, Radner and Shepp ${ }^{[17]}$, Paulsen and Gjessing $^{[18]}$, Højgaard and Taksar ${ }^{[19]}$, Taksar and Zhou $^{[20]}$, Asmussen and Taksar ${ }^{[21]}$, Asmussen et al. ${ }^{[22]}$, Taksar $^{[23]}$, Chouilli et al. ${ }^{[24]}$ and Paulsen ${ }^{[25]}$. None of the papers in the literature take advantage of the vast knowledge of Volterra integral equations as we do with this work. More to integral equations, we use numerical methods in this study. Before moving a step, we define a dividend and a barrier strategy.

Corresponding Author: Juma Kasozi, ${ }^{1}$ Department of Mathematics, Makerere University, P.O. Box 7062, Kampala, Uganda 
Definition 1: Dividends are taxable payments declared by the insurer's board of directors and given to shareholders out of the company's current or retained earnings.

Definition 2: A barrier strategy $b$ pays out any surplus above $b$ as dividends immediately so that the surplus is brought back to $b$. Below $b$, nothing is paid out.

\section{EXACT SOLUTION TO (1)}

To give a mathematical formulation of the models in this study, we take it that all processes and random variables are defined on the stochastic basis $\left(\Omega, f,\left\{f_{t}\right\}_{t \in \mathfrak{R}^{+}}, \mathrm{P}\right)$ satisfying the usual conditions, i.e. $f_{t}$ is rightly continuous and $\mathrm{P}-$ complete. Here, $\Omega$ is an abstract sample space whose elements is denoted as $\omega ; f$ is an $\sigma$-algebra on $\Omega ; \mathrm{P}$ is a probability measure and $\left\{f_{t}\right\}_{t \in \mathfrak{R}^{+}}$is a filtration. A filtration means an increasing and right continuous family of sub $\sigma$-algebras of $f$, that is, for $0 \leq s \leq t, f_{s} \subset f_{t} \subset f$ and $f_{t}=f_{t^{+}}=\cap_{s>t} f_{s}$.

Using Itô's formula, the infinitesimal generator for $Y$ is given by the following integral-differential operator:

$\operatorname{Ag}(y)=\operatorname{pg}^{\prime}(y)+\lambda \int_{0}^{\infty}(g(y-x)-g(y)) d F(x)$.

Now introduce a dividend barrier strategy $b$ so that in the infinitesimal time interval of length $d t$, the insurer pays the non-negative amount $d D_{t}^{b}$ in dividends. With such a strategy, (1) becomes:

$Y_{t+d t}^{b}=Y_{t}^{b}+p d t-d \sum_{i=1}^{N_{t}} S_{i}-d D_{t}^{b}, \quad Y_{0}^{b}=y>0$.

Let:

$V_{b}(y)=\mathrm{E}^{y}\left[\int_{0}^{T^{y}} e^{-\delta t} d D_{t}^{b}\right]$

denote the expected present value of dividend payments prior to ruin, that is, our dividend value function. Here, $\delta>0$ is the discount factor and time of ruin, $T_{y}^{b}=\inf \left\{t \geq 0: Y_{t}^{b}<0\right\}$.

In the said time interval $[t, t+d t]$, a claim occurs with probability $\lambda_{P} d t$. Hence, there will be no claim with probability $1-\lambda d t$. By law of total probability:

$$
\begin{aligned}
& \mathrm{V}_{\mathrm{b}}(\mathrm{y})=(1-\delta \mathrm{dt})(1-\lambda \mathrm{dt}) \mathrm{V}_{\mathrm{b}}(\mathrm{y}+\mathrm{pdt})+ \\
& \lambda \mathrm{dt} \int_{0}^{\infty} \mathrm{V}_{\mathrm{b}}(\mathrm{y}-\mathrm{s}) \mathrm{dF}(\mathrm{s}) ; \quad 0<\mathrm{y}<\mathrm{b}
\end{aligned}
$$

$$
\text { Substituting } \mathrm{V}+\mathrm{pdt}^{\prime} \text { for } \mathrm{V}_{\mathrm{b}}(\mathrm{y}+\mathrm{pdt}) \text { into }
$$
and simplifying gives:

$$
\mathrm{pV}^{\prime}-(\lambda+\delta) \mathrm{V}+\lambda \int_{0}^{\infty} \mathrm{V}_{\mathrm{b}}(\mathrm{y}-\mathrm{s}) \mathrm{dF}(\mathrm{s})=0
$$

Specifically, let the jumps be $\operatorname{Exp}(\alpha)$, so that the expectation $\mu=\frac{1}{\alpha}$. Then:

$\mathrm{pV}^{\prime}-(\lambda+\delta) \mathrm{V}+\alpha \lambda \int_{0}^{\mathrm{y}} \mathrm{V}_{\mathrm{b}}(\mathrm{y}-\mathrm{s}) \mathrm{e}^{-\alpha \mathrm{s}} \mathrm{ds}=0$

Put $x$ for $y-s$ into (6), partially differentiate and eliminate integral term to get:

$\mathrm{pV}^{\prime \prime}+(\alpha \mathrm{p}-\lambda-\delta) \mathrm{V}^{\prime}-\alpha \delta \mathrm{V}=0$

$\mathrm{V}_{\mathrm{b}}(0)=0, \quad \mathrm{~V}_{\mathrm{b}}^{\prime}(\mathrm{b})=1$

The solution to the specific case (7) which is also a solution for the general case (5), up to the barrier, is of the form ${ }^{[25]}$ :

$\mathrm{V}_{\mathrm{b}}(\mathrm{y})=\frac{\mathrm{g}(\mathrm{y})}{\mathrm{g}^{\prime}(\mathrm{b})}$

where, in the case of (7),

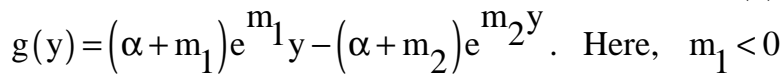
and $m_{2}>0$ are the roots of the characteristic equation corresponding to (7). Above the barrier, the solution becomes $V_{b}(y)=V_{b}(b)+y-b$. Infact $g(y)$ is any solution of $A g(y)=\delta g(y)$ where $A$ is given by (2). We observe that (5) takes this form.

Remark 1: For a given initial $y$, let $b^{*}$ denote the optimal value of $b$, thmaximizese value that maximises the expected present value of dividends. We see that this $b$ is the value that maximizes (8) thus minimizing $g^{\prime}(b)$. Hence $b^{*}$, is the solution of $g^{\prime \prime}\left(b^{*}\right)=0$. The $b$ that maximizes (8) is:

$\mathrm{b}^{*}=\frac{1}{\mathrm{~m}_{1}-\mathrm{m}_{2}} \log \frac{\mathrm{m}_{2}^{2}\left(\alpha+\mathrm{m}_{2}\right)}{\mathrm{m}_{1}^{2}\left(\alpha+\mathrm{m}_{1}\right)}$

else $b^{*}=y$.

All calculations were done on an IBM PC with 1024 MB RAM. The language was FORTRAN and we 
used DOUBLE PRECISION to get satisfactory accuracy. Of course slower programs like Splus, Matlab, Maple or Mathematica could have been used but at the expense of a considerably longer computing time. We obtained several valuable functions corresponding to the various chosen barriers. The optimal barrier $b^{*}=10.270110$ gives the $V^{*}$ (maximum dividend value function), all other value functions are less than this $V^{*}$ despite $b>b^{*}$. Later, we numerically compute the same dividend value functions using the block-by-block method developed in Paulsen et $a l^{[1]}$ and compare the results. These results and ideas herein will be handy in numerically computing value functions in the Cram'er-Lundberg model compounded by a constant force of interest.

\section{NUMERICAL SOLUTION TO (1)}

Here, our risk process is still (1) whereby $Y$ is the same process as the one described earlier 2 and $Y^{\infty}=Y$ thus:

$Y_{t}^{b}=y+p t-\sum_{i=1}^{N_{t}} S_{i}-D_{t}^{b}, \quad Y_{0}^{b}=y>0$

The dividend process $D^{b}$ is nondecreasing and continuous and $F(0)=0$. The total expected present value of dividends with discount factor $\delta$ and $Y_{0}^{b}=y$ is given by (3) where $T_{y}^{b}$ is ruin time, equal to infinity if ruin never occurs. Note that when $b<\infty$ then $\mathrm{P}\left(T_{y}^{b}<\infty\right)=1$ a.s. We use Theorem 1 below to find solutions to (3). Our strategy is to find the associated Volterra integral equation so that we use the methods in Paulsen et al. ${ }^{[1]}$.

Theorem 1: Assume model (9) and let $A$ be given by (2). Let $V_{b}(y)$ be bounded and twice continuously differentiable on $(0, b)$ with a bounded first derivative there. If $V_{b}(y)$ solves $A V_{b}(y)=\delta V_{b}(y)$ on $0<y<b$, together with the conditions:

$V_{b}(y)=0 \quad$ on $y<0, \quad V_{b}(0)=0$ if $\sigma_{P}^{2}>0$,

$V_{b}^{\prime}(b)=1, \quad V_{b}(y)=V_{b}(b)+y-b \quad$ on $y>b$.

Then $V_{b}(y)$ is given by (3).

Proof: Paulsen and Gjessing ${ }^{[18]}$.
Remark 2: In addition to Remark $1, b^{*}$ has geometric characterization. We have the expected present value of dividends:

$V_{b}(y)=\left\{\begin{array}{l}V_{b}(y), \quad 0 \leq y \leq b \\ V_{b}(b)+y-b, \quad y>b .\end{array}\right.$

At the junction $y=b, V_{b}(y)$ is continuous and has a continuous first derivative since $V_{b}^{\prime}(b)=1$. Using (8), $V_{b}^{\prime \prime}(b)=0$ is equivalent to $g^{\prime \prime}(b)=0$ which in turn means that $b=b^{*}$ since $g^{\prime \prime}\left(b^{*}\right)=0$. This geometric characterization of the optimal value is called the high contact condition in the finance literature and equivalently, the smooth pasting condition in the optimal stopping literature. For details, Dixit and Pindyck ${ }^{[26]}$.

Theorem 1 together with (2) gives the following version of (5):

$\mathrm{pV}_{\mathrm{b}}^{\prime}(\mathrm{y})+\lambda \int_{0}^{\mathrm{y}}\left(\mathrm{V}_{\mathrm{b}}(\mathrm{y}-\mathrm{x})-\mathrm{V}_{\mathrm{b}}(\mathrm{y})\right)$

Integrating $(10)$ on $[0, z]$ w.r.t. $y$ one concludes that:

$\mathrm{V}_{\mathrm{b}}(\mathrm{y})= \begin{cases}\mathrm{V}_{\mathrm{b}}(0)+\int_{0}^{\mathrm{y}}\left[\frac{\lambda+\delta}{\mathrm{p}}-\frac{\lambda}{\mathrm{p}} \mathrm{F}(\mathrm{y}-\mathrm{x})\right] \mathrm{V}_{\mathrm{b}}(\mathrm{x}) \mathrm{dx}, & 0 \leq \mathrm{y} \leq \mathrm{b},(11) \\ \mathrm{V}_{\mathrm{b}}(\mathrm{b})+\mathrm{y}-\mathrm{b}, & \mathrm{y}>\mathrm{b} .\end{cases}$

For $0 \leq y \leq b$, (11) is a linear Volterra integral equation of the second kind where the kernel is given by

$K(y, x)=\frac{\lambda}{p} F(y-x)-\frac{\lambda+\delta}{p}$ and the forcing function, $h(y)=V_{b}(0)$.

For any arbitrary starting $g(0), g(y)$ will be the $O\left(h^{4}\right)^{[1]}$ numerical solution from the block-by-block method. Then the solution to (11) is given by (8). To find $g^{\prime}(b)$, we use the approximation $g^{\prime}(b) \approx \lim _{h \rightarrow 0} \frac{g(b+h)-g(b-h)}{2 h}$, where $h$ is the grid size.

We have solved (11) for several values of $b$. At each run using a FORTRAN program that gives $O\left(h^{4}\right)$ solution to Volterra equations of the second kind (confer Paulsen et al. ${ }^{[1]}$ ), we have computed the $g^{\prime}(b)$. The results indicate that for any two barriers $b_{1}$ and $b_{2}, \quad 0<b_{1}<b_{2}<\infty, \quad g^{\prime}\left(b_{1}\right)>g^{\prime}\left(b_{2}\right)$. 
Eventually, some interval $\left\lfloor b_{1}, b_{2}\right\rfloor$ is given $g^{\prime}\left(b_{1}\right)<g^{\prime}\left(b_{2}\right)$ for the first time. This interval contains the optimal $b^{*}$ which gives the optimal value function $V_{b}\left(b^{*}\right)$. When $b=12, \quad g^{\prime}(12)>g^{\prime}(10)$ implying that the $b^{*}$ lies in the interval $[10,12]$.

To locate the actual $b^{*}$, we use our numerical solution of $A g(y)=\delta g(y)$ to calculate all the derivatives:

$$
g^{\prime}(i h) \approx \lim _{h \rightarrow 0} \frac{g((i+1) h)-g((i-1) h)}{2 h}
$$

and then locate the minimizing one. Then $b^{*}=i^{*} h$ for the minimizing $i^{*}$. The corresponding $g^{\prime}\left(b^{*}\right)$ value is then used to compute the numerical value function which is given by:

$$
V_{b}^{*}(y)= \begin{cases}\frac{g(y)}{g^{\prime}\left(b^{*}\right)}, & 0 \leq y \leq b^{*}, \\ \frac{g\left(b^{*}\right)}{g^{\prime}\left(b^{*}\right)}+y-b^{*}, & y>b^{*} .\end{cases}
$$

Table 1 shows the results. From the table, $i^{*}=$ 1027 implying that the $b^{*}=10.27$.

Finally, here, we compare the exact and numeric solutions. Let $V_{b}^{N}(y)$ be the numerically calculated value function when a numerical value of $b^{*}$, is used and $V_{b}^{A}(y)$ be the exact value function when the exact $b^{*}$ is used. Table 2 shows the absolute percentage relative error:

$$
D(y)=\left|100 . \frac{V_{b^{*}}^{N}(y)-V_{b^{*}}^{A}(y)}{V_{b^{*}}^{A}(y)}\right| .
$$

It is seen from Table 2 that the results are extremely good. This then gives us confidence to apply the numerical method to the model later.

\section{MODEL (1) COMPOUNDED BY A CONSTANT FORCE OF INTEREST}

The Cram'er-Lundberg model for the reserve $Y_{t}$ of an insurance company at time $t$ with a dividend process
$D^{b}$ is given by equation (9). A natural generalization of this model is to allow reserves to earn interest. If there is a positive constant force of interest $r$, the reserves now become

$$
\begin{aligned}
& \mathrm{Y}_{\mathrm{t}}^{\mathrm{b}}=\mathrm{y}+\mathrm{pt}+\mathrm{r} \int_{0}^{\mathrm{t}} \mathrm{Y}_{\mathrm{s}}^{\mathrm{b}} \mathrm{ds}-\sum_{\mathrm{i}=1}^{\mathrm{N}_{\mathrm{t}}} \mathrm{S}_{\mathrm{i}}-\mathrm{D}_{\mathrm{t}}^{\mathrm{b}} \\
& \mathrm{Y}_{0}^{\mathrm{b}}=\mathrm{y}>0
\end{aligned}
$$

Presence of a positive constant force of interest $r$ implies modifying (2) to a new infinitesimal generator for $Y$ now given by the following integro-differential operator:

$$
\begin{aligned}
& \operatorname{Ag}(y)=(r y+p) g^{\prime}(y)+ \\
& \lambda \int_{0}^{\infty}(g(y-x)-g(y)) d F(x)
\end{aligned}
$$

\begin{tabular}{|c|c|c|c|c|}
\hline \multicolumn{5}{|c|}{$\delta=0.1$} \\
\hline $\mathrm{i}$ & $y=i h$ & $\mathrm{~g}_{\mathrm{b}}(\mathrm{y})$ & $g^{\prime}(y)$ & $\mathrm{V}_{\mathrm{b}^{*}}(\mathrm{y})$ \\
\hline 0 & 0 & 5 & 0 & 0 \\
\hline 1 & 0.01 & 5.017489 & 1.7477949 & 5.3102001 \\
\hline 2 & 0.02 & 5.0349559 & 1.7455936 & 5.328686 \\
\hline 100 & 1 & 6.647174 & 1.5515921 & 7.034958 \\
\hline 200 & 2 & 8.1163843 & 1.3928355 & 8.5898793 \\
\hline 300 & 3 & 9.4437971 & 1.2669783 & 9.994731 \\
\hline 400 & 4 & 10.659443 & 1.1684631 & 11.281295 \\
\hline 500 & 5 & 11.788304 & 1.0927188 & 12.476012 \\
\hline 600 & 6 & 12.85121 & 1.0359868 & 13.600926 \\
\hline 700 & 7 & 13.865579 & 0.9951797 & 14.674472 \\
\hline 800 & 8 & 14.846027 & 0.9677631 & 15.712117 \\
\hline 1000 & 10 & 16.752547 & 0.9451688 & 17.72986 \\
\hline 1024 & 10.24 & 16.979344 & 0.9448811 & 17.969888 \\
\hline 1025 & 10.25 & 16.988792 & 0.9448792 & 17.979888 \\
\hline 1026 & 10.26 & 16.998241 & 0.944878 & 17.989888 \\
\hline 1027 & 10.27 & 17.00769 & 0.9448776 & 17.999888 \\
\hline 1028 & 10.28 & 17.017139 & 0.944878 & 18.009888 \\
\hline 1200 & 12 & 18.648711 & 0.955725 & 19.736642 \\
\hline 1400 & 14 & 20.591959 & 0.9911446 & 21.793257 \\
\hline 1600 & 16 & 22.626207 & 1.0460063 & 23.946179 \\
\hline 1800 & 18 & 24.786613 & 1.1168553 & 26.232618 \\
\hline 2000 & 20 & 27.102867 & 1.2015941 & 28.683999 \\
\hline
\end{tabular}

Then Theorem 1 together with (13) gives the integral-differential equation of Volterra type (for $0 \leq y \leq b)$ :

$$
\begin{gathered}
(\mathrm{ry}+\mathrm{p}) \mathrm{V}_{\mathrm{b}}^{\prime}(\mathrm{y})+\lambda \int_{0}^{\infty}\left(\mathrm{V}_{\mathrm{b}}(\mathrm{y}-\mathrm{x})-\mathrm{V}_{\mathrm{b}}(\mathrm{y})\right) \\
\mathrm{dF}(\mathrm{x})-\delta \mathrm{V}_{\mathrm{b}}(\mathrm{y})=0
\end{gathered}
$$

Table 1: Numerical $\mathrm{V}_{\mathrm{b}^{*}}(\mathrm{y})$ for $\operatorname{Exp}(0.5)$ jumps, $p=6, \lambda=2$ and

Table 2: Comparison of numeric and exact solutions: $\operatorname{Exp}(0.5)$ jumps, $\lambda=2, p=6, \delta=0.1$

\begin{tabular}{llll}
\hline $\mathrm{y}$ & $\mathrm{V}_{\mathrm{b}^{*}}^{\mathrm{N}}(\mathrm{y})$ & $\mathrm{V}_{\mathrm{b}^{*}}^{\mathrm{A}}(\mathrm{y})$ & $\mathrm{D}(\mathrm{y})$ \\
\hline 2 & 8.5898793 & 8.5898805 & $1.444 \mathrm{E}-05$ \\
4 & 11.281295 & 11.281297 & $1.427 \mathrm{E}-05$ \\
6 & 13.600926 & 13.600928 & $1.426 \mathrm{E}-05$ \\
8 & 15.712117 & 15.71212 & $1.426 \mathrm{E}-05$ \\
10 & 17.72986 & 17.729863 & $1.421 \mathrm{E}-05$ \\
12 & 19.729888 & 19.736645 & $1.419 \mathrm{E}-05$ \\
\hline
\end{tabular}


Theorem 2: The integral-differential equation (14) can be represented as the linear Volterra integral equation of the second kind:

$V_{b}(y)+\int_{0}^{y} K(y, x) V_{b}(x) d x=h(y)$,

where the kernel:

$K(y, x)=-\frac{(r+\lambda+\delta)-\lambda F(y-x)}{r y+p}$,

and the forcing function:

$$
h(y)=\frac{p V_{b}(0)}{r y+p} .
$$

Proof: Integrating (14) on $[0, z]$ w.r.t. $y$ and letting $v=$ $y-x$ one obtains:

$$
(\mathrm{rz}+\mathrm{p}) \mathrm{V}_{\mathrm{b}}(\mathrm{z})-\mathrm{pV}_{\mathrm{b}}(0)+\int_{0}^{\mathrm{z}}\left(\lambda_{\mathrm{p}} \mathrm{F}_{\mathrm{p}}(\mathrm{z}-\mathrm{v})-\left(\mathrm{r}+\lambda_{\mathrm{p}}+\delta\right)\right)
$$

Replacing $z$ with $y$ and $v$ with $x$ in (15) completes the proof.

We know from Theorem 1 that when $y>b, \quad V_{b}(y)=V_{b}(b)+y-b$. Hence, our value function takes the form:

$$
V_{b}(y)=\left\{\begin{array}{lc}
\frac{p V_{b}(0)}{r y+p}+\int_{0}^{y}\left(\frac{\left(r+\lambda_{p}+\delta\right)-\left(\lambda_{p} F_{p}(y-x)\right)}{r y+p}\right) V_{b}(x) d x, 0 \leq y \leq b, \\
V_{b}(b)+y-b, & y>b .
\end{array}\right.
$$

We carry with us the analysis, results and discussion from the previous section onto (16). The values of $g^{\prime}(b)$ decrease for $b=10,12,14$. When $b=$ 16, $g^{\prime}(16)>g^{\prime}(14)$ implying that the $b^{*}$ lies in the interval $[14,16]$.

To locate actual $b^{*}$, we again calculate all the derivatives using (12minimizing locate the minimising one. The results indicate that $i^{*}=1469$ thus giving $b^{*}=14.69$. Figure 1 is a graph of dividend value functions for some select barriers. The optimal dividend value function is the one corresponding to $b^{*}$.

When we expect heavy claims, then adequate probability should be assigned to the higher jumps by the distribution function $F$. Some of the distributions in this class are Lognormal, Pareto and Mixture of exponentials. These distributions are said to have heavy tails.

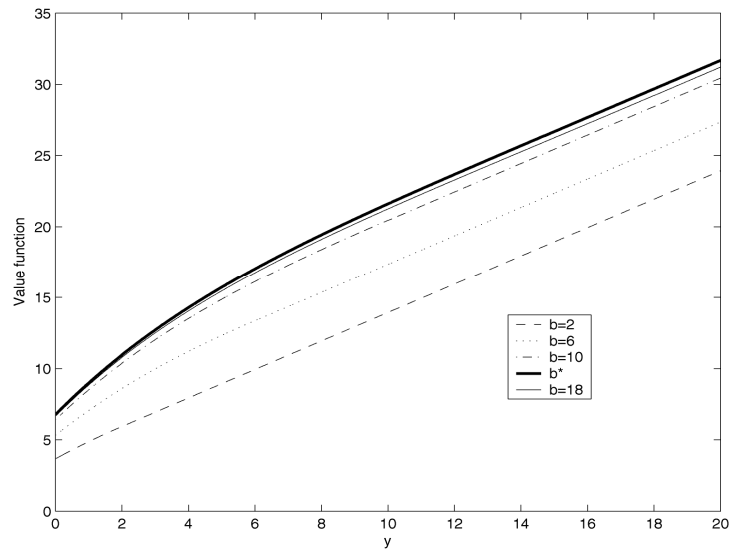

Fig. 1: Numeric value functions for $\operatorname{export}(0.5)$ jumps, $\lambda=2, p=6, r=0.05, \delta=0.1$

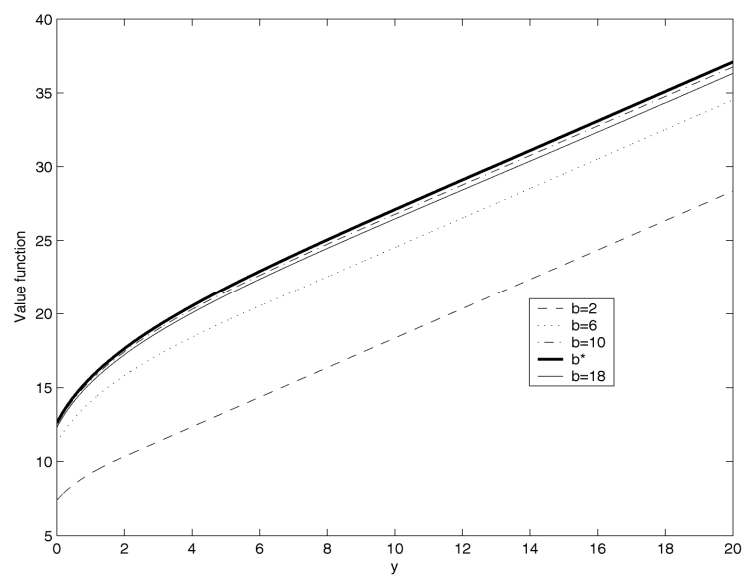

Fig. 2: Numeric value functions for a par (1.5) jumps, $\lambda=2, p=6, r=0.05, \delta=0.1$

We now report the results when the distribution for $S$ (generic for the $\mathrm{S}_{\mathrm{i}}$ ) is Pareto. The density function is given by:

$f(x)=(\alpha-1)^{\alpha} \alpha(\alpha-1+x)^{-(1+\alpha)}, \quad x>0, \alpha>1$.

Furthermore with this distribution:

$\bar{F}(x)=\left(\frac{\alpha-1}{\alpha-1+x}\right)^{\alpha}$

and

$\mathrm{E}(X)=\int_{0}^{\infty} \bar{F}(x) d x$.

If we set $\mathrm{E}(\mathrm{S})=2$, then $\alpha=1.5$. To locate actual $\mathrm{b}^{*}$, we again calculate all the derivatives using (12minimizing locate the minimising one. The results indicate that $i^{*}=1279$ thus giving $b *=12.79$. Figure 2 is a graph of dividend value functions for some select barriers. The optimal dividend value function is the one corresponding to $\mathrm{b}^{*}$. 


\section{CONCLUSION}

We numerically obtained maximal dividend function in the Cram'er-Lundberg model compounded by a constant force of interest using the block-by-block method and a barrier strategy. The results in Table 2 indicate its quality. An extension of this problem is to allow a diffusion in (1) thus:

$Y_{t}=p t+\sigma_{P} W_{P, t}-\sum_{i=1}^{N_{t}} S_{i} ; t \geq 0$.

With another diffusion, the return on investment process will no longer be $R_{t}=r t$ but:

$R_{t}=r t+\sigma_{R} W_{P, t} ; \quad t \geq 0$.

For a detailed description of such a model, see e.g. Paulsen et al. ${ }^{[1]}$. However, when there is a diffusion, band strategies might take an upper hand over barrier strategies and the mathematics changes accordingly.

\section{ACKNOWLEDGEMENT}

The authors extend their gratitude to NUFU project 33/02 and Carnegie Project under Carnegie/Mak/2002/0010 for the financial support and anonymous referees for the valuable comments.

\section{REFERENCES}

1. Paulsen, J., J. Kasozi and A. Steigen, 2005. A numerical method to find the probability of ultimate ruin in the classical risk model with stochastic return on investments. Insurance: Maths. and Econ., 36: 399-420.

2. Lundberg, F., 1903. I.Approximerad Framställning av Sannolikhetsfunktionen. II. Aterförsäkering av Kollektivrisker. Almqvist and Wiksell, Uppsala.

3. Cramér, H., 1930. On the Mathematical Theory of Risk. Skandia Jubilee Volume, Stockholm.

4. Cramér, H., 1954. On some questions connected with mathematical risk. Univ. California Publ. Statist., 2: 99-125.

5. Cramér, H., 1955. Collective Risk Theory. Skandia Jubilee Volume, Stockholm.

6. Miller, M.H. and F. Modigliani, 1961. Dividend policy, growth and the valuation of shares. J. Business, 34: 411-433.

7. De Finetti, B., 1957. Su un'impostazione alternativa dell teoria collectiva del rischio. Trans. 15th Intl. Cong. of Actuarities, New York, 2: 433443.

8. Seal, H.L., 1968. Stochastic Theory of a Risk Business. New York: Wiley.
9. Bühlmann, H., 1970. Mathematical methods in risk theory. Berlin Heidelberg New York: SpringerVerlag.

10. Gerber, H.U., 1973. Martingales in risk theory. Mitteilungen der Vereinigung Schweizer Versicherungsmatematiker, 73: 205-216.

11. Borch, K., 1974. The Mathematical Theory of Insurance. Lexington, MA: Lexington Books.

12. Borch, K., 1990. Economics of Insurance. Amsterdam: North-Holland.

13. Gerber, H.U., 1974. The dilemma between dividends and safety and a generalization of the Lundberg-Cramér formulas. Scand. Actuarial J., pp: 46-57.

14. Gerber, H.U., 1981. On the probability of ruin in the presence of a linear dividend barrier. Scand. Actuarial J., pp: 105-115.

15. Gerber, H.U., 1979. An Introduction to Mathematical Risk Theory. S.S. Huebner Foundation Monograpgh Series No. 8. Homewood, IL: Irwin.

16. Jeanblanc-Picqué, M. and A.N. Shiryaev, 1995. Optimization of the flow of dividends. Russian Math. Surveys, 50: 257-277.

17. Radner, R. and L. Shepp, 1996. Risk vs. Profit potential: A model for corporate strategy. J. Econ. Dynam. Control, 20: 1373-1393.

18. Paulsen, J. and H.K. Gjessing, 1997. Optimal choice of dividend barriers for a risk process with stochastic return on investments. Insurance: Math and Econ., 20: 215-223.

19. Højgaard, B. and M. Taksar, 1999. Controlling risk exposure and dividend payout schemes: Insurance company example. Math. Finance, 9: 153-182.

20. Taksar, M. and X.Y. Zhou, 1998. Optimize risk and dividend control of a company with a debt liability. Insurance: Math and Econ., 22: 105-122.

21. Asmussen, S. and M. Taksar, 1997. Controlled diffusion models for dividend payouts. Insurance: Maths and Econ., 20: 1-15.

22. Asmussen, S., B. Højgaard and M. Taksar, 2000. Optimal risk control and dividend distribution policies. Example of excess-of-loss reinsurance for an insurance corporation. Finance Stoch., 4: 299324.

23. Taksar, M., 2000. Optimal risk/dividend distribution models. Applications for insurance. Math. Methods Oper. Res., 1: 1-42.

24. Chouilli, T., M. Taksar and X.Y. Zhou, 2002. A di_usion model for optimal dividend distribution for a company with constraints on risk control. To appear Siam. J. Control Opt.

25. Paulsen, J., 2003. Optimal dividend payouts for diffusions with solvency constraints. Finance Stochast., 7: 457-473.

26. Dixit, A.K. and R.S. Pindyck, 1994. Investment under Uncertainty. First Edn. Princeton University Press. 